\title{
Manejo de recursos sólidos para la creación de ambientes óptimos de aprendizaje
}

\section{The management of Solid Resources for the creation of optimal learning environments}

DOI: http://dx.doi.org/10.17981/cultedusoc.9.3.2018.94

Artículo de investigación. Fecha de recepción: 15/06/2018. Fecha de aceptación: 27/11/2018

\author{
Ana Pino-Martínez; Ana Santander-Sanjuán; Antonio Cuello-Hernández; \\ Eduardo Peña-Núñez; Helena De Armas-Barrios; Karen Guerrero-Sarmiento; \\ Luis Meza-López; Luis Sarmiento-Vargas; Martha Onate-Quintero; \\ Noris Acosta-Martínez; Ramón Baena-Moya; Rita Ruiz-Torres; \\ Mario Sierra-Anaya y Shirle Luna-Molina ${ }^{1}$ \\ IED Macondo de la Sede Escuela Rural de varones José celestino Mutis (Colombia)
}

Para citar este artículo:

Pino-Martínez, A., Santander-Sanjuán, A., Cuello-Hernández, A., Peña-Núñez, E., De Armas-Barrios, H., Guerrero-Sarmiento, K., Meza-López, L., Sarmiento-Vargas, L., Oñate-Quintero, M., Acosta-Martínez, N., Baena-Moya, R., Ruiz-Torres, R., Sierra-Anaya, M. y Luna-Molina, S. (2018). Manejo de recursos sólidos para la creación de ambientes óptimos de aprendizaje. Cultura. Educación y Sociedad 9(3), 797-804. DOI: http://dx.doi.org/10.17981/cultedusoc.9.3.2018.94

\section{Resumen}

A nivel mundial existe una problemática de contaminación ambiental, generada por las actividades humanas, en gran medida por el manejo inapropiado de residuos sólidos, por lo cual se pretende que desde las instituciones educativas se generen estrategias pedagógicas que fomenten en los estudiantes actitudes pro ambientales, para preservar su entorno. El estudio tuvo como propósito fomentar el manejo de recursos sólidos para la creación de ambientes óptimos de aprendizaje. La metodología se orientó bajo el enfoque cualitativo, utilizando el tipo de investigación acción, desde un diseño descriptivo, exploratorio. Para la recolección de datos se utilizaron técnicas como; observación participante y diario de campo. Se escogió una muestra de veinticuatro (24) estudiantes desde primero de primaria hasta octavo de básica secundaria de la Institución Educativa Departamental Macondo de la Sede Escuela Rural de varones José celestino Mutis. Se evidencio un manejo integral de los residuos sólidos como parte del proceso de aprendizaje de la institución, llegando a conclusiones con una importancia en la trasformación de los residuos sólidos que mejoren el ambiente escolar.

Palabras clave: residuos sólidos, medio ambiente, reciclaje, basura, desorden.

\section{Abstract}

At a global level there is a problem of environmental pollution, generated by human activities, largely due to the improper handling of solid waste, which is why educational institutions are expected to generate pedagogical strategies that encourage students to adopt environmental attitudes, to preserve its environment. The purpose of the study was to promote the management of solid resources for the creation of optimal learning environments. The methodology was oriented under the qualitative approach, using the type of action research, from a descriptive, exploratory design. For the collection of data, techniques such as; participant observation and field diary. A sample of twenty-four (24) students from elementary school to eleventh secondary school was chosen from the Macondo Departmental Educational Institution of the Jose Celestino Mutis Rural School for males. An integral management of solid waste was evidenced as part of the learning process of the institution, reaching conclusions with an importance in the transformation of solid waste that improve the school environment.

Keywords: solid waste, environment, recycling, garbage, clutter.

1 Docentes IED Macondo de la Sede Escuela Rural de varones José celestino Mutis, Grupo de ingestigación Grupo. Mutisiny

- The author; licensee Universidad de la Costa - CUC

Cultura, Educación y Sociedad vol. 9 no. 3, pp. 797-804. Diciembre, 2018

Barranquilla. ISSN 2389-7724 Online 


\section{Introducción}

En Colombia, al igual que muchos otros países en el mundo, enfrenta grandes retos en el manejo integral de sus residuos sólidos en pertinencia al elevado índice de crecimiento demográfico e industrial y el cambio de habito en el consumo por parte de la colectividad, han generado una aceleración en el crecimiento residuos sólidos. En la actualidad, el interés en las cuestiones relacionadas con la protección del ambiente ha tenido su origen en los problemas del deterioro de los recursos naturales y los sistemas de comunicación y del incremento de la población.

Estos problemas han causado daños y perturbaciones en el ambiente, y representan un grave peligro para la salud y bienestar público (Morgado, Ramírez, Parody, Viloria, López y Kamatkar (2018), 2018; Albis, Ortiz y Martínez, 2017). Por otro lado, en los países en vías de desarrollo no son indiferentes ante estos problemas. La concentración de las actividades económicas en las grandes ciudades, es un factor preponderante en la generación de riesgos potenciales al ambiente, que pueden originar trastornos de carácter orgánico, fisiológico o social.

En efecto, las zonas urbanas presentan manifestaciones visibles de los problemas causados por una serie de agresores contaminantes de origen químico, físico y biológico, de no actuar con determinación pudiesen ser de mucho peligro, ya que afecta tanto a niños, jóvenes, adultos sin importar la raza, el género o la nacionalidad; la contaminación es cada vez mayor y poco o nada hacen los seres humanos para disminuir esta gran problemáticapara la comunidad en general.

El problema del medio ambiente es una dificultad a nivel global, debido a que los seres humanos no se concientizan sobre la necesidad imperante de cuidarlo siendo estos los principales beneficiados se convierten en su mayor detractor o destructor, este se ha convertido en un tema muy susceptible de ser estudiado ya que cada día se evidencia más las consecuencias de no cuidar el medio ambiente y los fuertes fenómenos naturales a causa de esto (Medina, 1999).

Por su parte, los procesos de extracción o de aprovechamiento de los recursos naturales, ejerce presiones excesivas sobre la propia naturaleza, no sólo derivadas en referencia a la transformación en bienes o por la disposición final de éstos cuando se convierten en residuos y que se vierten irresponsablemente en los suelos y cuerpos de agua. Las liberaciones de contaminantes al ambiente, han llegado a un extremo preocupante que compromete la calidad de vida y la sociedad, si no que conlleva a un agotamiento de los recursos naturales, que le mita un poco a la supervivencia en las generaciones presentes y futra, así como al propio crecimiento económico, por la forma en que han tenido lugar.

Por otra parte, en efecto, es corresponsabilidad de todos los actores educativos institucionales en la generación de dicho clima, que este debe existir una comunicación efectiva y dialógica dentro de la institución educativa, expandiendo la capacidad de escucha entre los directivos, los docentes y los estudiantes, prevaleciendo en este sentido unas relaciones cónsonas, con estilos característicos del docente de hoy, como es ser respetuosos acogedores y cálidos dentro del aula escolar, evitando los extremos de permisividad y autoritarismo. Lo que quiere decir que las normas y límites son acordados por la sociedad educativa, entendiéndose desde su sentido dinámico que todo el conjunto de actividades humanas tendientes a evitar la afectación, alteración o puesta 
en riesgo la existencia de la vida misma dependen de ella (Pereira, 2016).

\section{Elementos del manejo Integral de residuos sólidos Municipales}

Precisando de una vez, el objetivo fundamental del desarrollo sustentable de cualquier estrategia de manejo de residuos sólidos, es la matización mediante el beneficio de los recursos y la forma en reducir los impactos adversos al ambiente. De la misma manera, siempre habrá que hacer juicios de valor para reducir los impactos ambientales globales del sistema de manejo de residuos, permitiendo que al mismo la reducción de los costos, encontrando un punto de equilibrio para no generar debates y desvió de los objetivos trazados. Con base en la misma forma, se podrán tomar mejores decisiones en la medida que se cuente con datos para estimar unos costos reales en el tiempo trazado y determinar los impactos ambientales, lo cual puede generar nuevas ideas en el marco de los procesos de mejora continua.

Es decir que, un sistema de manejo adecuado de residuos sólidos, permite en cierto modo economía y ambientalmente proyectos netamente sustentables con un sentido integral en los términos y tiempos establecidos, que este mismo debe ser orientado a un mercado, flexible y capaz de manejar todos los tipos de residuos sólidos. La alternativa de centrarse en materiales específicos, ya sea porque son fácilmente reciclables, o por la percepción pública, puede ser en cierto modo pertinente a ser efectiva, mediante estrategias coherentes en donde se considere como factor fundamental saber concientizar a la colectividad en una mejor utilización de estos desechos sólidos.

Sobre la base de las consideraciones anteriores, en referencia a la generación de los residuos, se deben desarrollar ac- ciones que fomenten cambios en los patrones de producción, hábitos de consumo y diseño de productos, así como la innovación tecnológica y la investigación y desarrollo como herramienta eficaz que permita que las instituciones educativas existentes contribuyan en la participación de la comunidad involucrada, esto con la finalidad de evitar la contaminación que afecte el ecosistema. Todo ello, complementan los aspectos antes referidos, los que aparecen descritos en el cuadro que amplían y precisan lo que se concibe los aspectos a considerar en el diseño de las políticas de manejo ambientalmente adecuado de los residuos.

Además, es de suma importancia en reconocer desde una perspectiva crítica coherente aspectos centrales en la configuración en un manejo consolidado de los residuos solidados, de esta manera se deben manejar criterios unificados mediante una forma de trabajo basados en la cooperación como forma creativa para conservación del medio ambiente. En ese sentido, pensar desde un enfoque investigativo como parte fundamental en las corrientes educativas críticas y concientizadas en las comunidades, permite que la gente tenga una cultura enmarcada bajo principios que ayude a generar confianza en la comunidad en un sentido de nuevas formas de acumulación y dominación, esto significa que los elementos funcionales mejoran la eficiencia de los sistemas de manejo de los desechos sólidos en disposición en el medio ambiente con una sociedad organizada con principios de conservación (Ortega, 2009; Ramírez y Hugueth, 2017).

Dicho en otras palabras, la importancia de la investigación en la sociedad en cierto modo develan intereses presentes, lo que quiere decir que esta práctica devela una práctica que forja una mirada estrictamente critica, con una coheren- 
cia pertinente a los tiempos presentes, que obligan no solo a la denuncia, sino también a apropiarse de una realidad importante en ese sentido para construir propuestas que a la vez promuevan su apropiación práctica crítica, y sirvan para modernizar, y transformar bajo un contexto, epistemológico, y redirigir sus escenarios de poder construir subjetividades y ciudadanías que reconociendo el lugar de lo local se abran a un tiempoespacio global y a las nuevas mediaciones científico y tecnológicas de este tiempo para hacer concreta la construcción sociedades más democráticas, justas y humanas, así como la necesidad de un planteamiento sobre ella en cualquier proyecto emancipador.

De acuerdo con los razonamientos que se han venido realizando, la realidad del principio de la educación popular se conoce con un sentido en transformarla desde un horizonte ético que busca develar y enfrentar la segregación, la exclusión y formas de control y dominación que se produce por las múltiples manifestaciones del poder en nuestra sociedad: en referencia a políticas, económicas, sociales, étnicas pertinente al caso, con un sentido enfocado y derivado de un conocimiento, de subjetividades, de género y en el ejercicio de la práctica educativa y pedagógica; construir un espíritu de emancipación humana, para no desarrollar procesos de poder que controlan ni dominan, ni permitir que otros lo realicen en su propia vida (Torres, 2007; Ramírez y Ampudia, 2018).

Es evidente entonces, que las sociedades actuales, juegan un papel preponderante en pertinencia al educador, donde el mismo permite ser enseñante-aprendices, este mismo haciendo un reconocimiento de manera tal manera como se forma. En este sentido, el papel del formando es activo y también forma a sus adultos acompañantes, a la vez que va aprendiendo, gestándose unas relaciones educativas desde lo diferente en la interculturalidad (De Souza, 2001; Ramírez, Avendaño, Aleman, Lizarazo, Ramírez y Cardona, 2018), en el respeto y el reconocimiento de los aportes de cada uno. Por ello en la IEP, maestro y maestra se convierten en acompañantes co-investigadores, transformando su rol tradicional y reconociéndose como aprendientes en el proceso.

\section{Metodología}

Se realizó un estudio de tipo cualitativo, utilizando el modelo de investigación acción, desde un diseño descriptivo, exploratorio. La investigación cualitativa busca conocer e interpretar la realidad de los participantes a través de sus propias experiencias, entregando una información subjetiva del fenómeno de estudio. (Hernández, Fernández, y Baptista, 2010). Por su parte el modelo de investigación acción plantea una producción de conocimiento basado en la reflexión de los participantes, teniendo en cuenta la participación activa de maestros en el proceso de enseñanza y acompañamiento tecnológico, guiando al estudiante en su proceso de investigación. Generando nuevo saber y conocimiento sobre una realidad determinada en un proceso de aprendizaje colaborativo.

Los estudios con un diseño descriptivo están encaminados a especificar las características de la población sujeto de estudio o los fenómenos que sean sometidos a análisis. Bajo un estudio de tipo exploratorio, debido a que no se han realizado estudios previos en la institución. (Hernández, Fernández, y Baptista, 2010). Se escogió una muestra de veinticuatro (24) estudiantes desde primero de primaria hasta octavo de básica 
secundaria de la Institución Educativa Departamental Macondo de la Sede Escuela Rural de varones José celestino Mutis ubicada en el municipio de Zona Bananera, Magdalena.

Para la recogida de datos se utilizó la observación participante y el diario de campo, estas son técnicas de recolección cualitativas que implican que el docente tome un rol activo en el proceso de investigación, donde no solo debe estar como espectador del proceso de implementación, sino que también debe participar del mismo, registrando cada elemento observado con relación a la variable de estudio. En el diario de campo se registran y sistematizan los elementos obtenidos de la observación participante donde el contacto con las personas del contexto, modulan las reflexiones y conclusiones que se pueden extraer.

La metodología del proyecto de investigación se realizó a partir de los recorridos de las trayectorias de indagación, basados en la integración de la IEP al aula, articulado al plan de estudios, transversalizando así la educación ambiental a las diversas asignaturas, lo anterior con la finalidad de generar una trasformación social y educativa a través del aprendizaje en contexto, involucrando intereses, motivaciones y realidades de los estudiantes para generar conocimiento científico.

\section{Resultados}

El eje de esta propuesta está fundamentado en saberes propios de una cultura establecidas por un conocimiento enmarcada en un horizonte investigativo, de hecho esta planteada por los diferentes grupos de jóvenes, que en cierto sentido busca a través de ella la unidad y la relación de saberes y conocimientos, con un criterio basados en propuestas metodológica que realiza mediante el reconocimiento social de los diferentes actores, que a su vez ponen de manifiesto diferentes concepciones, que podrán tramitar reconociendo la visibilidad de múltiples métodos investigativos, en coherencia con el tipo de problema. Obteniendo los siguientes resultados:

1. Concientización de los estudiantes frente al cuidado del medio ambiente.

2. Decoración de la institución con mensajes alusivos al reciclaje.

3. Creación de un centro de acopio para los residuos sólidos desechados en la escuela.

4. Clasificación de los residuos recolectados.

5. Creación de elementos decorativos.

6. Optimización del ambiente escolar por parte de docentes y estudiantes.

7. Sistematización de los procesos de recolección de residuos.

Se realizaron jornadas de aseo en la institución, con la participación de los distintos grupos de estudiantes y el acompañamiento de los docentes. Aactualmente, se encuentra en el proceso de reciclar todo el residuo sólido plástico como son los envases de agua y bebidas azucaradas, los cuales serán reutilizados en la elaboración de materas y canecas para el depósito de los residuos sólidos generados en la institución.

En tal sentido, el pilar fundamental para la ejecución del proyecto, ha sido el trabajo que los educadores de las diferentes áreas que realizan durante las clases, donde buscan que los estudiantes se apropien del espacio institucional en el cuidado y conservación del ambiente, dentro y fuera del aula de clase, la idea es poder concientizar a todos los actores de la comunidad pertinente a llevar un resultado positivo de una forma integradora y justa. 


\section{Conclusiones}

Son muchas las expectativas en cuanto a la aplicación y ejecución del programa llegando a las siguientes conclusiones:

a. Se demuestra el sentido de pertenencia con la institución.

b. Se apropiaron los estudiantes de los conceptos como reciclaje, medio ambiente, residuos sólidos y buen clima escolar

c. La optimización del ambiente escolar se vea reflejada en los buenos procesos que se llevan a cabo en la escuela

d. Embellecimiento de la sede educativa con el Mantenimiento de toda la institución por parte de docentes y estudiantes.

A partir de lo anterior se puede concluir que las malas prácticas ambientales que se venían practicando en la IED mejoraron sustancialmente con la realización de la anterior propuesta. Al realizar actividades con docentes, padres de familia y estudiantes se integraron los miembros de la comunidad educativa involucrando a estos en la solución de los problemas que se presentaban solucionándolos de forma grata y satisfactoria. Por esto se puede decir que el reciclaje es la mejor manera de sacarle provecho a los materiales utilizados para crear otros nuevos materiales, con esta práctica se puede contribuir a que todo nuestro alrededor esté más limpio y de esta manera tener un clima saludable para el aprendizaje de los educandos.

\section{Referencias}

Albis, A., Ortiz, J. y Martínez, J. (2017). Remoción de cromo hexavalente de soluciones acuosas usando cáscara de yuca (Manihot esculenta): Experimentos en columna. INGE CUC, 13(1), 42 52. https://doi.org/10.17981/ingecuc.13. 1.2017 .04
Careaga J. (2013). Manejo y reciclaje de los residuos de envases y embalajes. Serie Monografías No. 6. SEDESOL. Instituto Nacional de Ecología.

De souza, J. (2001). Atualidade de Paulo Freire. Contribuição ao debate sobre a educação na diversidade cultural. Recife: Edições Bagaço.

Fals-Borda, O. (1984). Resistencia en el San Jorge. Historia doble de la Costa. Tomo III. Bogotá, D.C.: Carlos Valencia Editores.

Freire, P. (1986). Hacia una pedagogía de la pregunta. Conversaciones con Antonio Faúndez. Buenos Aires: La Aurora.

Herrera, B., Guerrero, H. y Ramírez, R. (2018). Investigación como estrategia pedagógica: Una mirada desde la educación, escuela y transformación de la comunidad global. Barranquilla: Universitaria de la Costa..

Hernández, R., Fernández, C. y Baptista, P. (2010). Metodología de la investigación. México, D.F.: Editorial Mc Graw Hill.

Mariño, G. (2010). El diálogo en la educación de jóvenes y adultos. Dos propuestas pedagógicas para implementarlo. En, El taller dialógico/la recuperación de experiencias laborales. Bogotá, D.C.: OEI.

Maturana, H. (1998). Emoções e linguagem na educação e na política. Belo Horizonte: UFMG.

Medina, M. (1999). Reciclaje de desechos sólidos en América Latina. Frontera Norte, 11(21). 7-31. http:// dx.doi.org/10.17428/rfn.v11i21. 1411 
Medina, J, Jiménez, I, Aguirre, I, Vallejo, S, Tobón, R, y Rocha, M. (2009). Minimización y manejo ambiental de los residuos sólidos. México, D.F.: Subsecretaría de Gestión para la Protección Ambiental-SEMARNAT.

Mejía, M. y Manjarrés M. (2010). Las pedagogías fundadas en la investigación. Búsquedas en la reconfiguración de la educación. +Revista Internacional Magisterio, 42(1). $16-26$.

Mejía, M. (2011b). Las escuelas de la globalización. El conflicto por su reconfiguración. Bogotá, D.C.: Desde Abajo.

Mignolo, W. (2003). Capitalismo y geopolitica del conocimiento. Buenos Aires: Paidós.

Morgado, W., Ramírez M., Parody, A., Viloria, A., López, M. y Kamatkar, S. (2018). Concentrations and Size Distributions of Fungal Bioaerosols in a Municipal Landfill. In: Tan Y., Shi Y., Tang Q. (eds) Data Mining and Big Data. DMBD 2018. Lecture Notes in Computer Science, vol 10943. Springer, Cham.

Ortega, P. (2009). Sujetos y prácticas de la pedagogía crítica. Bogotá, D.C.: El Búho.

Pereira, M. (2016). Hacia la construcción de un derecho energético ambiental como disciplina autónoma. Jurídicas CUC, 12(1), 177-204. http://dx.doi.org/10.17981/juridcuc.12.1.2016.10

Pérez, T. (2011). El clima escolar, factor clave en la educación de calidad. Cartagena de Indias: Fondo Editorial del Caribe.
Porlán, R. (1995). Constructivismo y escuela: hacia un modelo de enseñanza aprendizaje basado en la investigación. Sevilla: Diada.

Pozo, J. y. (2006). Aprender y ensenar ciencia. Madrid: Morata.

Ramírez, R. y Hugueth, A. (2017). Modelo de comunicación productiva para las organizaciones de salud pública en Venezuela. Revista Opción, 33(83). 305-335. Disponible en http://produccioncientificaluz.org/index.php/opcion/article/ view/23118

Ramírez, R. y Ampudia, D. (2018). Factores de Competitividad Empresarial en el Sector Comercial. Revista Electrónica de Ciencia y Tecnología del Instituto Universitario de Tecnología de Maracaibo (RECITIUTM), 4(1). 16-32. Disponible en http://recitiutm.iutm. edu.ve/index.php/recitiutm/article/view/130

Ramírez, R., Avendaño, I., Aleman, L., Lizarazo, C., Ramírez, R. y Cardona, Y. (2018). Principles of social responsibility for the strategic management of the talent human public health organizations. Revista Espacios, 39(37). 22-27. Disponible en http://www. revistaes pacios . com/ a18v39n37/18393722.html

República de Colombia. Ministerio de Educación. (2015). Ruta de reflexion y mejoramiento pedagogico "Siempre Dia E" (Vol. 4). Bogota, D.C.: Mineducación. Recuperado de http://aprende.colombiaaprende.edu.co/sites/default/ files/naspublic/Guia\%204_ Ciclo\%203_V4ok.pdf 
República de Colombia. Ministerio de Educación. (2002). Por el cual se reglamenta la Ley 142 de 1994, la Ley 632 de 2000 y la Ley 689 de 2001, en relación con la prestación del servicio público de aseo, y el Decreto Ley 2811 de 1974 y la Ley 99 de 1993 en relación con la Gestión Integral de Residuos Sólidos. [Decreto 1713]. Diario Oficial: 44893. Recuperado de http://www.alcaldiabogota.gov.co/sisjur/normas/ Norma1.jsp?i $=5542$
Torres, A. (2007). La educación popular. Trayectoria y actualidad. Bogotá, D.C.: El Búho.

Vigotsky, L. (1979). El desarrollo de los procesos psíquicos superiores. Barcelona: Crítica 